\title{
Towards Quantitative SPECT: Error Estimation of SPECT OSEM with 3D Resolution Recovery, Attenuation Correction and Scatter Correction
}

\author{
J. Zeintl, Student Member, IEEE, A. H. Vija, Member, IEEE, A. Yahil, Member, IEEE, X. Ding, Member, IEEE, J. \\ Hornegger, Member, IEEE, and T. Kuwert
}

\begin{abstract}
In this study we systematically investigate biases relevant to quantitative SPECT if OSEM with isotropic (3D) depth dependent resolution recovery (OSEM-3D), attenuation and scatter correction is used. We focus on the dependencies of activity estimation errors on the projection operator, structure size, pixel size, count density and reconstruction parameters. We use Tc-99m to establish a base line. Four Siemens low energy collimators (Low Energy Ultra High Resolution, Low Energy High Resolution, Low Energy All Purpose, Low Energy High Sensitivity) with geometric resolution between $4.4 \mathrm{~mm}$ and 13.1 $\mathrm{mm}$ at $10 \mathrm{~cm}$ distance and sensitivity between $100 \mathrm{cpm} / \mu \mathrm{Ci}$ and $1020 \mathrm{cpm} / \mu \mathrm{Ci}$ are tested with simulations of spheres with diameters between $9.8 \mathrm{~mm}$ and $168 \mathrm{~mm}$ in background. Pixel sizes and total counts are varied between $2.4 \mathrm{~mm}$ and $9.6 \mathrm{~mm}$ and 0.125 and 32 million counts. Images are reconstructed with OSEM-3D (Flash3D) with attenuation and scatter correction. Emission recovery is quantitatively measured for different reconstruction parameter settings. In addition, physical measurements of standard quality control phantoms are performed using an actual SPECT/CT system (Symbia ${ }^{\circledR}$ T6). Cross calibration of the imaging system with a well counter and results from simulations are used to quantitatively estimate the true activity concentration in the physical phantoms. Results show variations of emission recovery between $13.8 \%$ and $104.5 \%$ depending on sphere volume and number of OSEM-3D updates. After correction for the emission recovery errors and cross calibration of the imaging system the errors in absolute quantitation using the physical sphere phantom are between $+0.01 \pm 0.61 \%$ for the largest $(16 \mathrm{ml})$ and $-5.87 \pm 1.00 \%$ for the smallest $(0.5 \mathrm{ml})$ sphere. As a conclusion, the emission recovery varies over a wide range and is highly dependent on imaging parameters when using OSEM-3D reconstruction. Accurate quantitation in phantoms is possible given that errors at the specific imaging operation point can be estimated. In a clinical setup this is a nontrivial task, and perhaps too cumbersome for routine clinical use.
\end{abstract}

Manuscript received November 14, 2008. Asterisk indicates corresponding author.

*Johannes Zeintl is a PhD student with the University of ErlangenNuremberg, Institute of Pattern Recognition, Erlangen, Germany. (e-mail: Johannes.Zeintl@uk-erlangen.de).

A. Hans Vija, Ding Xinhong are with Siemens Medical Solution USA, Inc., Molecular Imaging, Hoffman Estates, IL 60192, USA.

Amos Yahil is with Image Recon LLC, Stony Brook, NY, USA.

Joachim Hornegger is with the University of Erlangen-Nuremberg, Institute of Pattern Recognition, Erlangen, Germany.

Torsten Kuwert is with the University of Erlangen-Nuremberg, Clinic of Nuclear Medicine.

\section{INTRODUCTION}

T $\mathrm{N}$ clinical SPECT imaging iterative reconstruction techniques are now a common alternative to filtered backprojection [5]. Ordered subset expectation maximization (OSEM) is often the iterative method of choice ([3], 10]). The advantage of iterative methods is the ability to correct for image degradations introduced by imaging physics such as depth dependent blur, photon attenuation, and scatter. It was shown that these corrections minimize errors for absolute quantitation in SPECT imaging ([2], [9], [11], [12]). Active research is conducted to improve correction techniques for quantitation in SPECT ([16] - [20]) and to evaluate common reconstruction methods and establish practical baselines ([4], [6], [13], [15]). However, less attention was paid to the non-stationary behavior of OSEM in terms of quantitation and the dependency of quantitation errors on imaging parameters. In this work we systematically investigate biases relevant to quantitative SPECT if OSEM with isotropic (3D) depth dependent resolution recovery (OSEM-3D), attenuation and scatter correction is used. We focus on the dependencies of activity estimation errors on the projection operator, structure size, pixel size, count density, and reconstruction parameters. We use the obtained results to correct for the non-stationarity of OSEM in physical phantom experiments performed on a cross-calibrated SPECT/CT system.

\section{MATERIAL AND METHODS}

Three steps were performed to derive absolute values for activity concentrations from reconstructed images of actual measured data:

A: Estimation of emission recovery errors for various imaging parameter sets using simulations.

B: Cross calibration of the SPECT/CT imaging system with the well counter.

C: Appliance of correction factors derived from A and B to measured phantom data. 


\section{A. Simulations of imaging system}

Quasi-analytical simulations (voxel size: $0.6 \mathrm{~mm}$ ) are performed by modeling the projection operator in 3D according to the detector and collimator specifications of the Siemens Symbia T-series gamma cameras with the parameters listed in Table I, and assuming a perfect collimator. Poisson noise is added to the generated projection data. Photon attenuation in the object is accounted for and a derived $\mu$-map is used for attenuation correction. We assume an acquisition with perfect scatter rejection of $140 \mathrm{keV}$ (Tc-99m) photons.

TABLE I

COLLIMATOR SPECIFICATIONS OF FOUR SiEMENS LOW ENERGY COLLIMATORS

\begin{tabular}{|c|c|c|c|c|}
\hline & $\begin{array}{c}\text { Acceptance } \\
\text { Angle (rad) }\end{array}$ & $\begin{array}{c}\text { Sensitivity } \\
\mathbf{( c p m} / \boldsymbol{\mu C i})\end{array}$ & $\begin{array}{c}\text { Hole Length } \\
\mathbf{( m m})\end{array}$ & $\begin{array}{c}\text { Geometric } \\
\text { Resolution } \mathbf{a} \\
\mathbf{1 0 c m}(\mathbf{m m})\end{array}$ \\
\hline LEHS & 0.115194 & 1020 & 24.05 & 13.4 \\
LEAP & 0.064973 & 330 & 24.05 & 7.6 \\
LEHR & 0.049514 & 202 & 24.05 & 5.9 \\
LEUHR & 0.034632 & 100 & 35.8 & 4.4 \\
\hline
\end{tabular}

LEHS: Low Energy High Sensitivity, LEAP: Low Energy All Purpose, LEHR: Low Energy High Resolution, LEUHR: Low Energy Ultra High Resolution

Hot spheres with diameters between $9.8 \mathrm{~mm}$ and $168 \mathrm{~mm}$ in a warm cylindrical background (Sphere to background ratio 10:1) are simulated. Pixel size and total counts are varied between $2.4 \mathrm{~mm}$ and $9.6 \mathrm{~mm}$ and 0.125 and 32 million counts. Image reconstruction is performed using MLEM/OSEM with isotropic resolution recovery (Flash3D) and attenuation correction. Fig. 1 shows example images of simulated and reconstructed spheres.

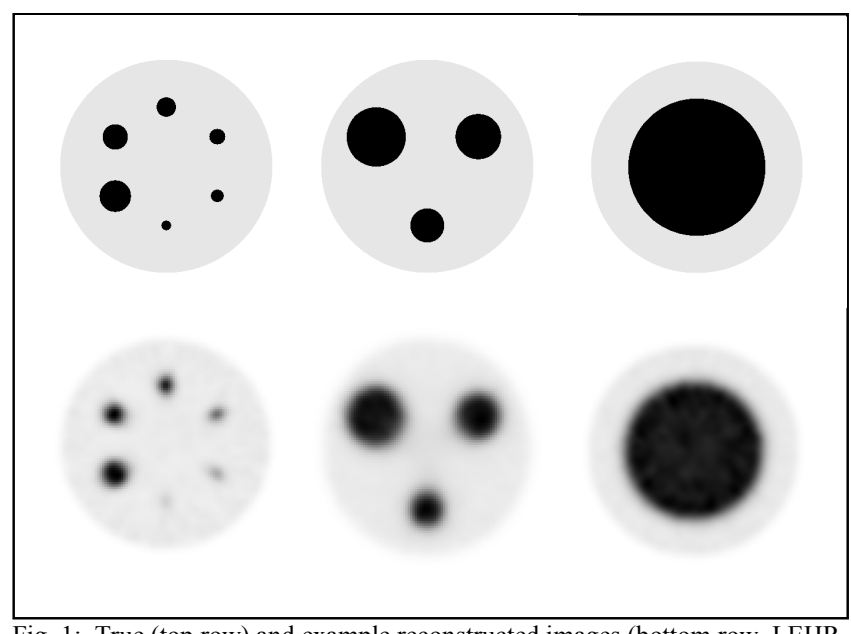

Fig. 1: True (top row) and example reconstructed images (bottom row, LEHR collimation, $2.4 \mathrm{~mm}$ voxel, 32 OSEM updates) of the simulated spheres of different diameters in a $10 \%$ background.

Images are analyzed in terms of emission recovery. The emission recovery coefficient is defined as:

$$
C_{E}(j, i)=\frac{\text { Measured Emission }(j, i)}{\text { True Emission }(j)},
$$

for a given object $j$ at a given imaging parameter set $i$. The boundaries of the target object to be measured are derived from the true high resolution image (Fig. 1 top row). Partial volume effects, specifically spill-in and spill-out at the object boundaries due to finite pixel size, are compensated for by measuring the loss of emission in the simulation using different pixel sizes.

\section{B. Cross Calibration of the Imaging System}

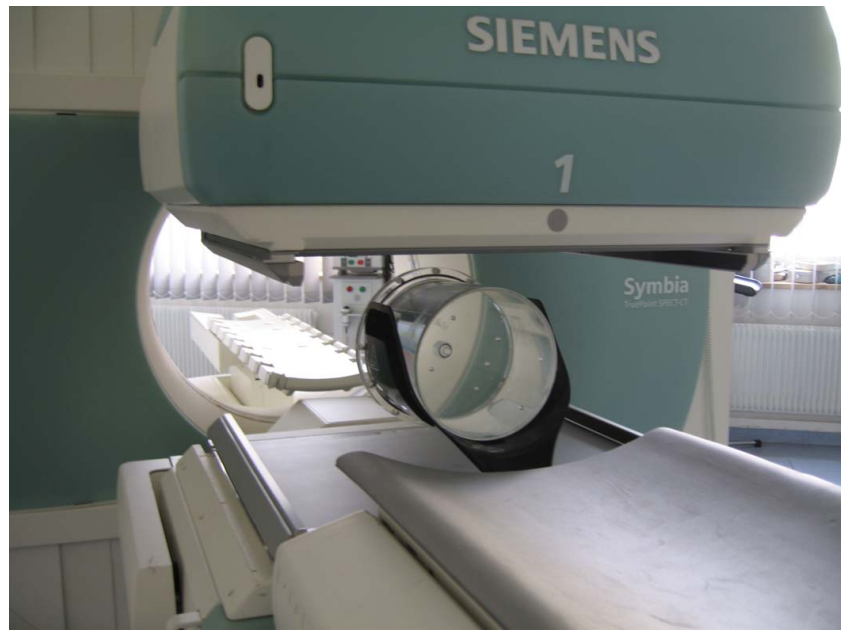

Fig. 2: Acquisition setup for the cross calibration of the imaging system. A large cylindrical phantom is filled uniformly with a known activity concentration derived from a well counter and acquired in the SPECT/CT imaging system.

The cross calibration of the SPECT/CT system is done using a large cylindrical phantom uniformly filled with water and a known activity concentration of Tc-99m measured in a well counter (see Fig. 2). 50 million total counts are collected in a 360 degree acquisition range, 120 projections, and a 150 $\mathrm{mm}$ detector radius from the center of rotation. A dual energy window was used for the acquisition of the photo peak and the lower scatter $(15 \%$ window width, respectively). An attenuation map was generated from a CT scan of the phantom. Data are reconstructed using OSEM with isotropic beam modeling (Flash3D) and scatter and attenuation correction.

To calculate the system volume sensitivity a large volume of interest $(>3000 \mathrm{ml})$ is drawn and the count rate per volume is derived. The decay corrected count rate is calculated as follows:

$$
\hat{R}=R \times \exp \left(\frac{T-T_{0}}{T_{1 / 2}} \ln 2\right) \times\left(\frac{\ln 2}{T_{1 / 2}}\right)\left(1-\exp \left(-\frac{t}{T_{1 / 2}} \ln 2\right)\right)^{-1}
$$

Where $R$ is the count rate derived from the reconstructed image, $T$ is the start time of the acquisition, $T_{0}$ is the time of the activity calibration, $T_{1 / 2}$ is the half time of the isotope, and $t$ is the time duration of the acquisition.

The system volume sensitivity is then: 


$$
S_{\text {Vol }}=\frac{\hat{R} / t_{\text {Dwell }}}{c_{A}}
$$

Where $t_{D \text { well }}$ is the total dwell time of the acquisition $\left(t_{D \text { well }}=\right.$ $t$ in continuous acquisition mode) and $c_{A}$ is the actual activity concentration in the phantom measured by the well counter.

\section{Appliance of corrections on real phantom data}

To evaluate the quantitative accuracy of the SPECT/CT imaging system a standard quality control sphere phantom (Data Spectrum, Hillsborough, NC) is acquired. The activity concentration is $19.6 \mu \mathrm{Ci} / \mathrm{ml}(725 \mathrm{kBq} / \mathrm{ml})$ in the spheres and $1.7 \mu \mathrm{Ci} / \mathrm{ml}(63 \mathrm{kBq} / \mathrm{ml})$ in the background, resulting in a concentration ratio of approx. 12:1. The phantom is acquired in the center of the field of view using a $150 \mathrm{~mm}$ detector radius of rotation over a $360^{\circ}$ scan range. LEHR collimation is used with a $2.4 \mathrm{~mm}$ pixel size. The total number of counts is 24 million.

The data are reconstructed with Flash3D with scatter and CT based attenuation correction. Volumes of interest are drawn manually using the CT boundaries of the fused SPECT/CT image (see Fig. 3). The absolute activity concentration for a given object size $j$ is calculated by

$$
\hat{c}_{A}(j)=\frac{R_{\text {VOI }}(j) / t_{\text {Dwell }}}{S_{\text {Vol }} C_{E}\left(j, i^{\prime}\right)},
$$

where $R_{V O I}$ is the count rate per volume in the drawn volume of interest and $i$ ' the specific imaging parameter set used.

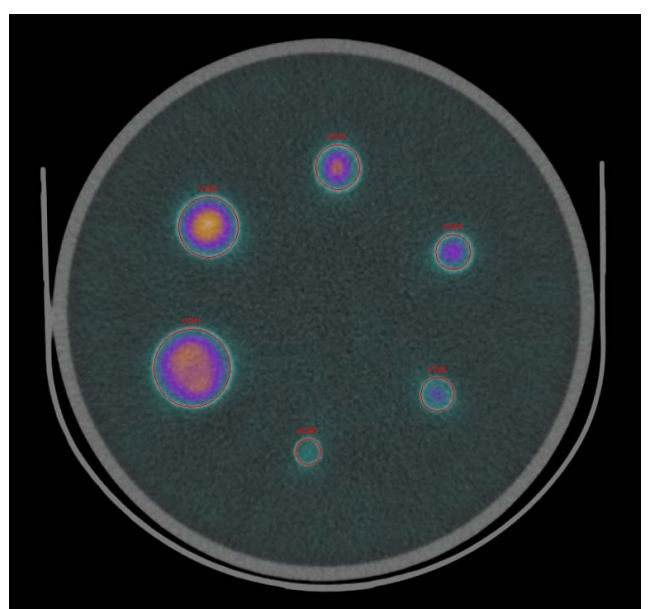

Fig. 3: Reconstructed image of the sphere phantom fused with the CT image (LEHR collimation, $2.4 \mathrm{~mm}$ voxel, 32 OSEM updates). Volumes of interest were drawn manually using the $\mathrm{CT}$ boundaries.

\section{RESULTS}

\section{A. Simulation Results}

In Fig. 4 the loss of emission recovery due to spill over at the object boundaries is shown for the different object and voxel sizes used. The values are derived from the simulation tool when a target to background ratio of 10:1 is used. In subsequent results these values are applied as a postprocessing step after reconstruction to compensate for the effect by adding the respective value to the emission recovery coefficients measured in the simulations.

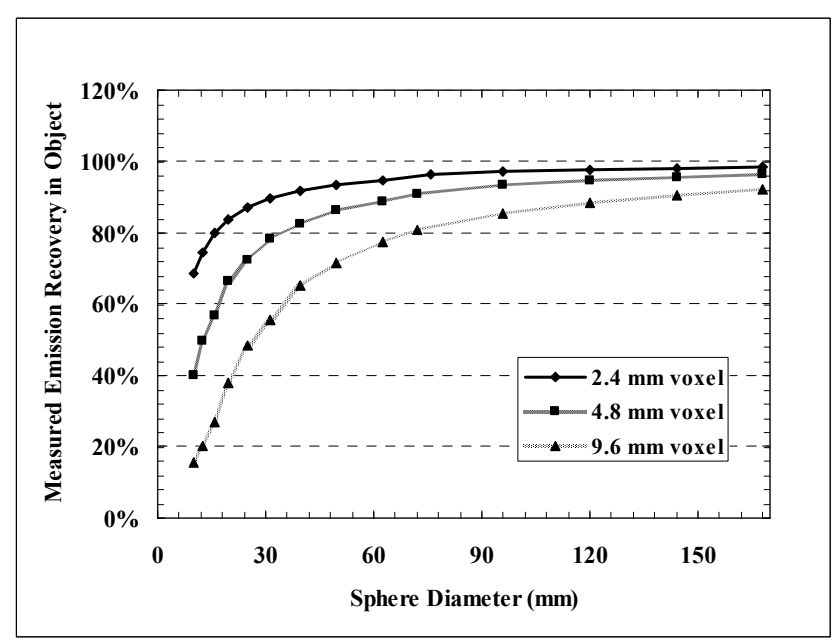

Fig. 4: The effect of spill over at object boundaries on emission recovery due to finite voxel size for different object and voxel sizes (target to background ratio is $10: 1)$.

Fig. 5 gives an overview of the emission recovery coefficients for different object sizes, number of OSEM updates, and voxel sizes used. Results are shown for LEHR collimation and 2 million total counts. For small object and voxel sizes $(2.4 \mathrm{~mm}, 4.8 \mathrm{~mm})$ the over shooting characteristic of OSEM at high numbers of updates is visible. In general the emission recovery coefficient is highly dependent on the number of OSEM updates especially for object sizes below 3 times the system resolution. This dependency at small object sizes is more pronounced for finer data sampling (smaller voxels).

The dependency of emission recovery on the projection operator is shown in Fig. 6. The recovery coefficient for LEUHR, LEAP, and LEHS collimation is shown using 2.4 mm voxel size, and 1 million, 4.5 million, and 10 million total counts for LEUHR, LEAP, and LEHS collimation, respectively. At small object sizes the effect of the beam geometry on the emission recovery is noticeable and results in lower values for broader beam collimators. 

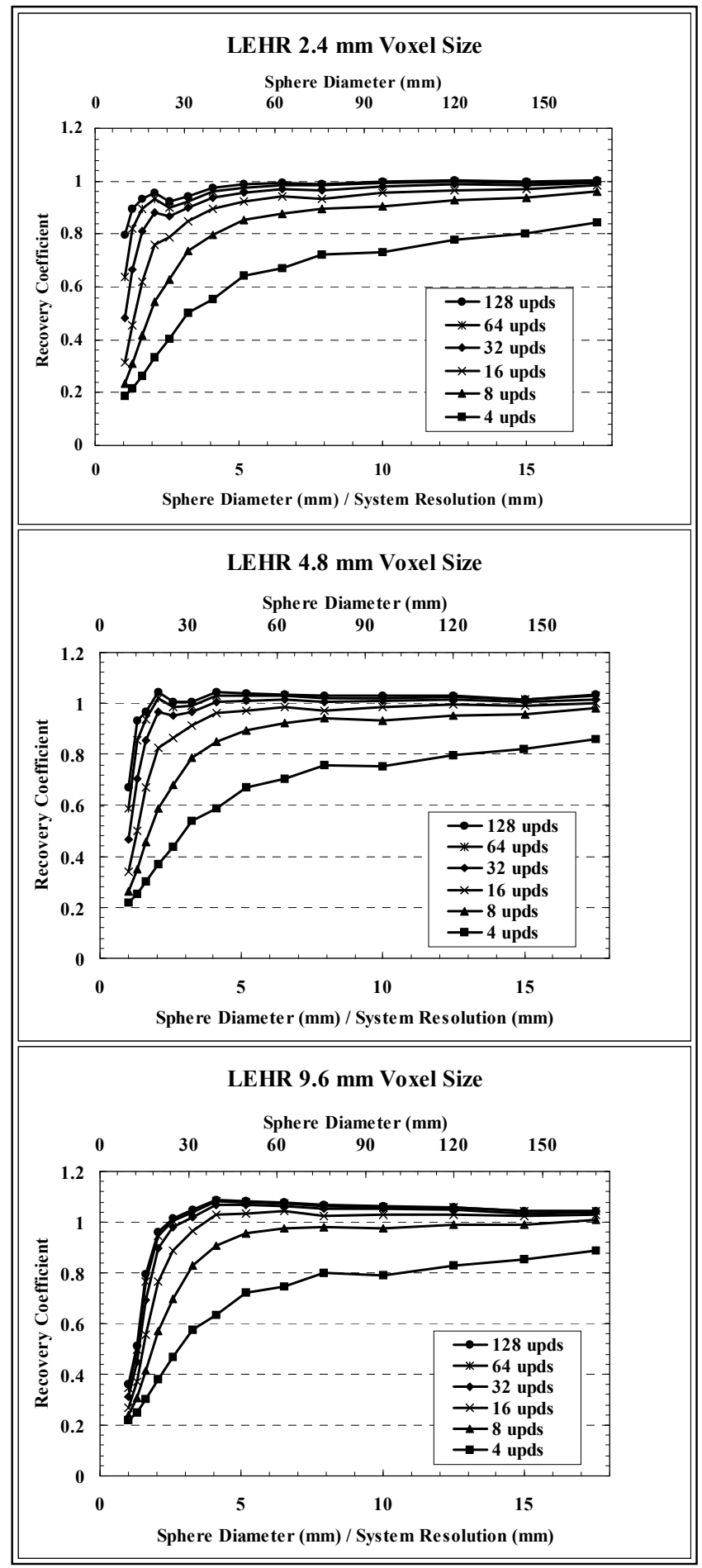

Fig. 5: Emission recovery coefficients for different object sizes, number of OSEM updates, and voxel sizes using LEHR collimation and 2 million total counts. Values are corrected for spill over effects at the object boundaries using results of Fig. 4. Each data point is obtained using the average value of 5 independent realizations.

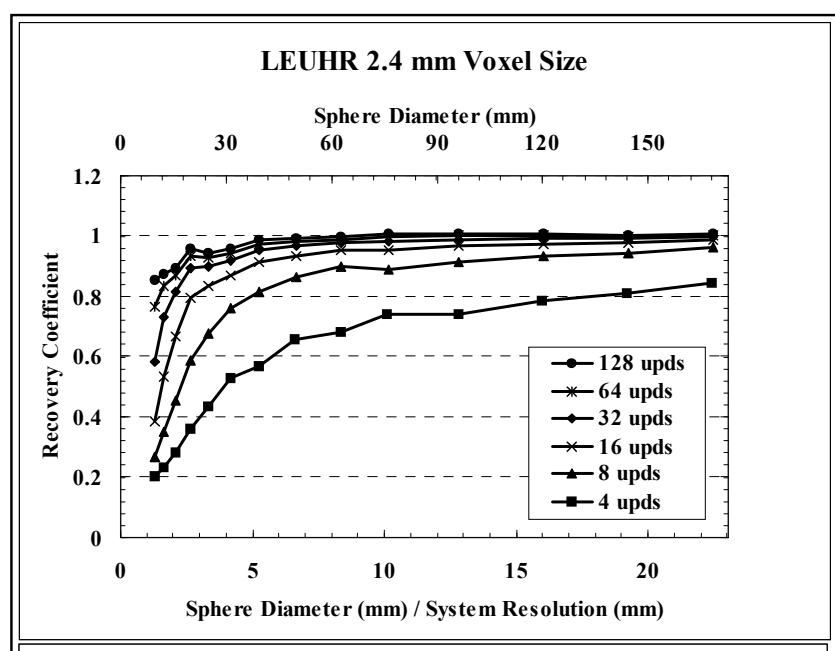

LEAP 2.4 mm Voxel Size
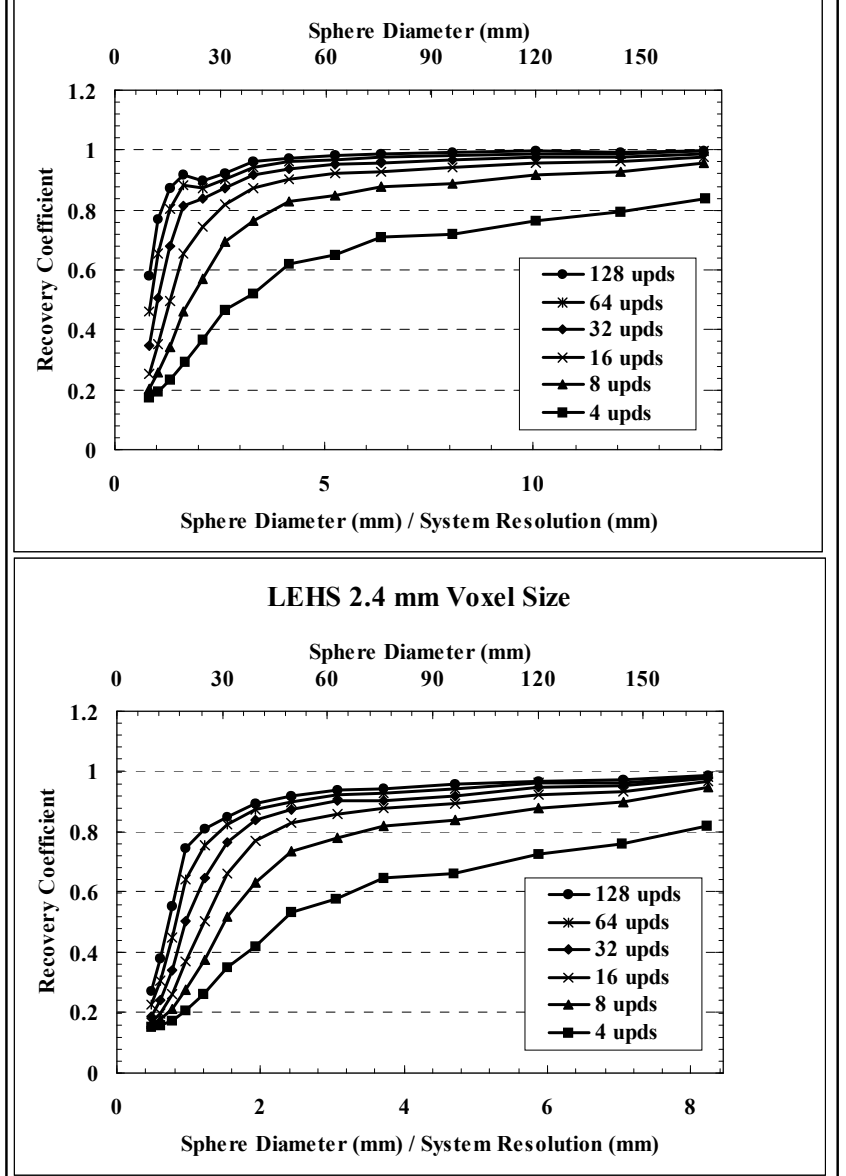

Fig. 6: Emission recovery coefficients for different object sizes, number of OSEM updates, and collimation schemes using $2.4 \mathrm{~mm}$ voxels and 1 million, 4.5 million, and 10 million total counts for LEUHR, LEAP, and LEHS collimation, respectively. Values are corrected for spill over effects at the object boundaries using results of Fig. 4. Each data point is obtained using the average value of 5 independent realizations. 
The dependency of total counts on the emission recovery is shown in Fig. 7. Beyond 3 times the system resolution the standard deviation of the recovery coefficient is below 0.0035 for all count levels tested. Below this point standard deviations are between 0.0056 and 0.0233 for $16 \mathrm{ml}$ and $0.5 \mathrm{ml}$ spheres, respectively.

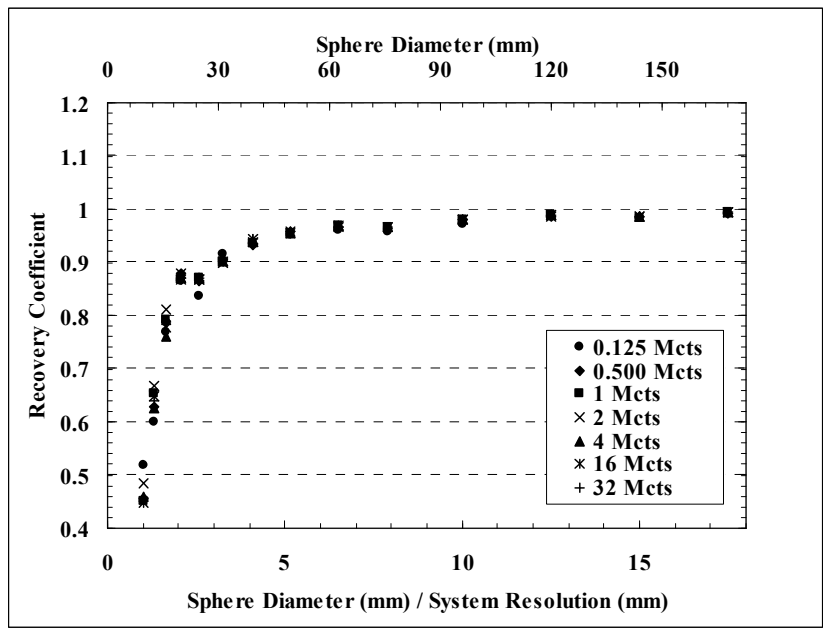

Fig. 7: Emission recovery coefficients for different total count levels using LEHR collimation, $2.4 \mathrm{~mm}$ voxel size and 32 OSEM updates. Each data point is obtained using the average value of 5 independent realizations.

\section{B. Quantitative Results from Phantom Experiment}

Using the simulated recovery coefficient for the specific operation point of the phantom experiment (Section II C) which is between 0.485 and 0.898 for the smallest and the largest sphere respectively and applying Equation 4, the absolute activity concentrations are calculated for all 6 spheres. The results are summarized in Table II showing absolute quantification errors between $-5.87 \%$ and $0.01 \%$ for the smallest and largest sphere, respectively.

TABLE II

RESULTS FROM PHANTOM EXPERIMENT

\begin{tabular}{|c|c|c|c|c|c|}
\hline $\begin{array}{c}\text { Sphere } \\
\text { Volume } \\
\text { (ml) }\end{array}$ & $\begin{array}{c}\text { Activity } \\
\text { Concentratio } \\
\text { n }(\mu \mathrm{Ci} / \mathbf{m l})\end{array}$ & $\begin{array}{l}\text { VOI Mean } \\
\text { Volume } \\
(\mathrm{ml})\end{array}$ & $\begin{array}{c}\text { Image Based } \\
\text { Calculated } \\
\text { Mean } \\
\text { Activity } \\
\text { Concentratio } \\
\text { n }(\mu \mathrm{Ci} / \mathrm{ml})\end{array}$ & $\begin{array}{c}\text { Activity } \\
\text { Concentratio } \\
\text { n Standard } \\
\text { Deviation** } \\
(\mu \mathrm{Ci} / \mathrm{ml})\end{array}$ & $\begin{array}{c}\text { Difference - } \\
\text { Reference } \\
\text { vs. } \\
\text { Calculated } \\
(\%)\end{array}$ \\
\hline 16 & & 16.3 & 19.59 & 0.12 & $-0.01 \pm 0.61$ \\
\hline 8 & & 8.2 & 20.03 & 0.13 & $+2.25 \pm 0.66$ \\
\hline 4 & 19.6 & 4.1 & 20.11 & 0.29 & $+2.62 \pm 1.48$ \\
\hline 2 & & 2.0 & 19.60 & 0.22 & $+0.02 \pm 1.15$ \\
\hline 1 & & 1.1 & 18.65 & 0.34 & $-4.81 \pm 1.73$ \\
\hline 0.5 & & 0.6 & 18.44 & 0.19 & $-5.87 \pm 1.00$ \\
\hline
\end{tabular}

*according to manufacturer specifications and well counter measurements

**manual determination of VOIs by 2 operators, 2 repeats each

\section{Discussion}

The object evaluated in the phantom experiment geometrically correlates well to the objects in the simulation, i.e. object sizes and positions are similar. In addition, the scanner geometry including e.g. detector orbit and radius, of simulation and experiment is kept identical. Thus, dependencies of geometric conditions on the emission recovery are disregarded.

The method used to simulate the imaging system only takes the primary photons of $140 \mathrm{keV}$ into account, neglecting both septal penetration as well as object and collimator scattering. These effects are present in the real data even though corrected using the standard techniques provided by the reconstruction software package. These inconsistencies might introduce errors.

During the cross calibration step for obtaining the system volume sensitivity additional errors are introduced due to both systematic and statistical errors of physical volume and activity measurements.

In the phantom experiment the object boundaries are drawn manually resulting in variable size and shape even though fused CT data is used. To create reproducible results and for use in clinical workflows automatic or semi automatic segmentation tools are preferable.

The developed procedure shows promising results for absolute quantitation in SPECT. However, a careful analysis incorporating all the various sources of error is required, in order to make this procedure more universally valid.

\section{CONCLUSION}

Results from simulations show that the emission recovery varies over a wide range and is highly dependent on imaging parameters when using OSEM-3D reconstruction. Highest variations are in the clinical relevant domain below three times the imaging system resolution. Accurate quantitation in phantoms is possible given that errors at the specific imaging operation point can be estimated. In a clinical setup this is a nontrivial task, and perhaps too cumbersome for routine clinical use.

\section{ACKNOWLEDGMENTS}

The authors would like to thank Ron Malmin and Manjit Ray for valuable discussions and the Systems Test team at Hoffman Estates for their help during system quality control and acquisition of the data.

\section{REFERENCES}

[1] H. H. Barrett, and e. al., "Noise Properties of the EM algorithm: I. Theory," Physics in Medicine and Biology, vol. 39, pp. 833-846, 1994.

[2] D. R. Gilland, R. J. Jaszczak, J. E. Bowsher et al., "Quantitative SPECT Brain Imaging: Effects of Attenuation and Detector Response," Nuclear Science, IEEE Transactions on, vol. 40, no. 3, pp. 295-299, 1993.

[3] H. M. Hudson, and R. S. Larkin, "Accelerated Image Reconstruction using Ordered Subsets of Projection Data," Medical Imaging, IEEE Transactions on, pp. 100-108, 1994.

[4] C. Kamphuis, F. J. Beekman, and M. A. Viergever, "Evaluation of OSEM vs. ML-EM for 1D, 2D and fully 3D SPECT reconstruction," 
Nuclear Science, IEEE Transactions on, vol. 43, no. 3, pp. 2018-2024, 1996.

[5] F. C. Klocke, and e. al., "ACC/AHA/ASNC Guidelines for the Clinical Use of Cardiac Radionuclide Imaging," ACC/AHA/ASNC Practice Guidelines, 2003.

[6] T. R. Miller, and J. W. Wallis, "Clinically Important Characteristics of Maximum-Likelihood Reconstruction," Journal of Nuclear Medicine, vol. 33, pp. 1678-1684, 1992.

[7] J. Nuyts, "On Estimating the Variance of Smoothed MLEM Images," Nuclear Science, IEEE Transactions on, vol. 49, no. 3, pp. 714-721, 2002.

[8] P. H. Pretorius, M. A. King, T.-S. Pan et al., "Reducing the influence of the partial volume effect on SPECT activity quantitation with 3D modelling of spatial resolution in iterative reconstruction," Physics in Medicine and Biology, vol. 43, pp. 407-420, 1998.

[9] M. S. Rosenthal, J. Cullom, W. Hawkins et al., "Quantitative SPECT Imaging: A Review and Recommendations by the Focus Commitee of the Society of Nuclear Medicine Computer and Instrumentation Council," Journal of Nuclear Medicine, vol. 36, pp. 1489-1513, 1995.

[10] L. A. Shepp, and Y. Vardi, "Maximum Likelihood Reconstruction for Emission Tomography," Medical Imaging, IEEE Transactions on, vol. MI-1, no. 2, pp. 113-122, 1982.

[11] B. M. W. Tsui, E. C. Frey, X. Zhao et al., "The importance and implementation of accurate 3D compensation methods for quantitative SPECT," Physics in Medicine and Biology, vol. 39, pp. 509-530, 1994.

[12] B. M. W. Tsui, X. Zhao, E. C. Frey et al., "Quantitative Single-Photon Emission Computed Tomography: Basics and Clinical Considerations," Seminars in Nuclear Medicine, vol. 14, no. 1, pp. 38-65, 1994.

[13] A. H. Vija, E. G. Hawman, and J. C. Engdahl, "Analysis of a SPECT OSEM Reconstruction Method with 3D Beam Modeling and Optional Attenuation Correction: Phantom Studies," IEEE NSS/MIC Conference Record, 2003.

[14] D. W. Wilson, and H. H. Barrett, "The Effects of Incorrect Modeling on Noise and Resolution Properties of ML-EM Images," Nuclear Science, IEEE Transactions on, vol. 49, no. 3, pp. 768-773, 2002.

[15] T. Yokoi, and e. al., "Performance Evaluation of OSEM reconstruction algorithm incorporating three-dimensional distance-dependent resolution compensation for brain SPECT: A simulation study," Annuals of Nuclear Medicine, vol. 16, no. 1, pp. 11-18, 2002.

[16] S. Shcherbinin and A. Celler, "An investigation of iterative reconstructions in quantitative SPECT", Journal of Physics: Conference Series 124 (2008)

[17] S. Shcherbinin and A. Celler, "Non-iterative quantitative SPECT reconstructions with a reduced size system matrix", 2007 IEEE Nuclear Science Symposium Conference Record, M13-325

[18] E. Vandervoort, A. Celler, and R. Harrop, "Implementation of an iterative scatter correction, the influence of attenuation map quality and their effect on absolute quantitation in SPECT", Phys. Med. Biol. 52 (2007), 1527-1545

[19] S. Liu and T.H. Farncombe, "Collimator-Detector Response Compensation in Quantitative SPECT Reconstruction", 2007 IEEE Nuclear Science Symposium Conference Record, M19-327

[20] K. Willowson, et al., "Quantitative SPECT reconstruction using CTderived corrections", Phys. Med. Biol. 53 (2008), 3099-3112 